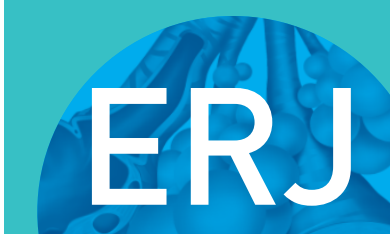

open research

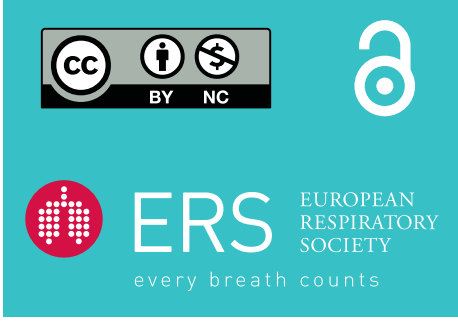

\section{Rapid and sustained airway control in metastatic malignant melanoma:} a case report

To the Editor:

A 70-year-old female presented with a 3-week history of a productive cough, progressive dyspnoea and small-volume haemoptysis. She was a lifelong nonsmoker with a past medical history of malignant melanoma of the left shoulder, which was treated with wide local excision 8 years prior to this presentation. This was a pT2N0M0 lesion with a Breslow thickness of $2 \mathrm{~mm}$, negative resection margins and sentinel lymph nodes. She did not take any regular medications, had no drug allergies, and was a nun and a retired nurse with a history of occupational exposure to tuberculosis.

She was transferred from a regional hospital to our centre with a provisional diagnosis of non-small cell lung cancer (NSCLC) from endobronchial biopsies performed at the referring institution. Transfer was made for further staging with endobronchial ultrasound (EBUS) and for central airway management if required. On admission, peripheral oxygen saturations were $90 \%$ on room air and clinical examination revealed reduced air entry in the right lung. A computed tomography scan of the thorax demonstrated a 40-mm subcarinal mass with invasion of the right main bronchus. An elective flexible bronchoscopy demonstrated an exophytic tumour almost completely occluding the right mainstem bronchus (RMB) and an EBUS confirmed a large subcarinal mass, which was also sampled (figure 1a). Further biopsies were obtained for immunohistochemistry (IHC) and a rigid bronchoscopy was planned for the next working day, 3 days later. On the day of the rigid bronchoscopy, pre-procedure, she deteriorated clinically with increasing respiratory distress associated with a fall in peripheral oxygen saturation to $70 \%$ on room air and a portable chest radiograph revealed complete collapse of the right lung. A rigid bronchoscopy under general anaesthesia was expedited and mechanical debulking was performed, using the distal end of the rigid bronchoscope to core the intraluminal component of the obstructing tumour in the RMB. Intraprocedural argon plasma coagulation (APC) was employed as an adjuvant thermal therapy to facilitate tumour cell ablation and haemostasis. This procedure was well tolerated, and resulted in substantial clinical and radiological improvement.

IHC from endobronchial specimens were negative for thyroid transcription factor 1 and cytokeratin 7, and positive for the melanoma markers Melan-A and S100. In the context of a history of previous malignant melanoma, a final diagnosis of recurrent malignant melanoma with endobronchial metastasis (EBM) was established. There was no evidence of melanoma recurrence at the primary cutaneous or other sites. A gene mutation was identified in the BRAF codon 600 site in exon 15, and BRAF and MEK inhibitors, dabrafenib and trametinib, were commenced with adjuvant consolidation radiotherapy to the mediastinum. Surveillance bronchoscopy 15 months later showed complete resolution of endobronchial disease (figure $1 \mathrm{~b}$ ). This patient remained clinically stable on maintenance therapy 5 years following disease recurrence and 13 years since the initial diagnosis of malignant melanoma.

This is an interesting case of an EBM secondary to recurrent malignant melanoma, and highlights important concepts regarding the aetiology, classification and management of central airway obstruction (CAO).

@ERSpublications

Endobronchial metastasis from extrapulmonary malignancies are rare and include malignant melanoma. Cases that are complicated by central airway obstruction should follow a patientcentred approach, guided by patient and tumour characteristics. http://bit.ly/32baZvo

Cite this article as: Cullivan S, Bruzzi J, McHale T, et al. Rapid and sustained airway control in metastatic malignant melanoma: a case report. ERJ Open Res 2019; 5: 00050-2019 [https://doi.org/ 10.1183/23120541.00050-2019].

Copyright $\odot$ ERS 2019. This article is open access and distributed under the terms of the Creative Commons Attribution NonCommercial Licence 4.0. 

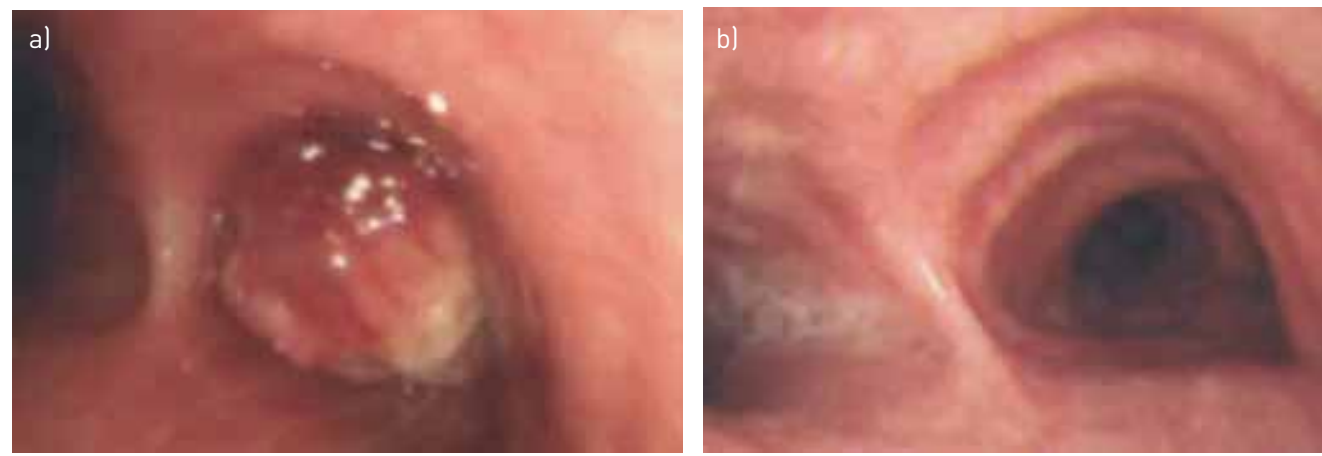

FIGURE 1 a) Bronchoscopy of 70-year-old female presenting with dyspnoea, a productive cough and haemoptysis demonstrates a large necrotic lesion completely obstructing the right main bronchus. b) Interval bronchoscopy the same patient following mechanical debulking and targetted immunotherapy for an obstructing endobronchial metastasis in the proximal right main bronchus from recurrent malignant melanoma shows complete resolution of endobronchial disease.

Malignant CAO has been reported to complicate $\sim 30 \%$ of lung cancer cases [1]. Causes of CAO include primary and metastatic cancer from both intrathoracic and extrathoracic sites [2]. The clinical presentation varies, and depends on patient and tumour characteristics, including the degree of endoluminal obstruction and underlying physiological reserve. As highlighted in this case report, patients frequently present with nonspecific symptoms of dyspnoea, cough and recurrent pulmonary infections. If the physician does not maintain a high index of suspicion, this can lead to diagnostic delays, as alternative diagnoses such as asthma, COPD or vocal cord dysfunction are considered first. Additionally, initial chest radiographs are frequently unremarkable, which can further hamper the diagnosis [3].

Malignant CAO is classified as extraluminal (extrinsic), endoluminal (intrinsic) or combined intrinsic and extrinsic disease (mixed) [2]. This case is an example of mixed CAO as there was additional subcarinal adenopathy associated with the endoluminal disease at the RMB. KIRYU et al. [4] suggested that endotracheal and EBMs from nonpulmonary malignancies are further classified into four groups to aid the description of developmental patterns. EBMs from extrapulmonary malignancies are rare, and are most commonly associated with breast cancer, colorectal cancer and renal cell carcinoma. Malignant melanoma accounts for a small proportion of cases at $\sim 4 \%$ [5]. While EBMs are rare, intraparenchymal pulmonary metastases are common, presumably as the entire cardiac output is pumped through the pulmonary vasculature, while the airways are supplied by an alternative system via the bronchial arteries. Autopsy series have demonstrated subclinical metastatic melanoma in almost every organ [6]. The development of macrometastases reflects a complex interplay between melanoma cells, local immunity and site-specific adaptations. The concept of dormancy, the extent of tumour dissemination and the role of immune surveillance are highlighted by cases of fatal malignant melanoma in immunosuppressed transplant recipients who inadvertently received organs from donors with a distant history of malignant melanoma $[6,7]$.

Due to significant clinical heterogeneity associated with $\mathrm{CAO}$, the subsequent management should be individualised, and should consider both patient factors such as performance status and prognosis, and tumour characteristics including location and type of obstruction. In this case, mechanical and thermal therapies were used to obtain immediate recanalisation of the obstructed airway. The distal end of the rigid bronchoscope was used to "mechanically" core the obstructing tumour and complementary APC was subsequently employed as a noncontact thermal therapy to the tumour bed. This procedure was well tolerated, and potential complications such as thermal injury, endobronchial perforation or gas embolism were avoided by maintaining an inspiratory oxygen fraction $<40 \%$ during APC, with a distance of $\geqslant 5 \mathrm{~mm}$, power $<30 \mathrm{~W}$ and maximum duration of $10 \mathrm{~s}$ in any location. Subsequent thoracic radiation and systemic targetted immunotherapy were performed in this case. This combination of therapies resulted in a lasting patency without recurrence at this site. This was an appropriate treatment modality in this case as the patient had an excellent performance status, and the clinician knew that there was functional distal pulmonary parenchyma with only a short interval of lung collapse. In addition, the resultant debulked tumour allowed for adequate material for further IHC and mutational analysis, which is becoming increasing important in tailoring therapy for patients with specific malignancies including malignant melanoma. In this case, we describe the role of combined mechanical and thermal therapies to achieve successful recanalisation as these are the preferred procedures in our unit. Other methods have been well described in the literature, and include hot and cold modalities that can result in immediate or delayed debulking. These can include laser therapy, cryotherapy, brachytherapy, and endoluminal silicone and 
metallic stents. Stent insertion is particularly useful for extrinsic CAO or significant airway calibre compromise despite maximal debulking [2].

No individual method has been shown to be superior to another in obtaining good results, and the choice of technique is often based on individual expertise and availability of equipment within units. In this case, rigid bronchoscopy with mechanical debulking was combined with subsequent combined targetted therapy and mediastinal radiation, reinforcing the concept that endoluminal debulking should not be viewed solely as a palliative procedure and can be used as part of multimodal therapy for synergistic treatment effects.

This is an interesting case of an atypical presentation of metastatic malignant melanoma with CAO. This case highlights the role of the interventionalist as part of a multimodal diagnostic and therapeutic algorithm, not just for lung cancer, but for all endobronchial tumours. It also underscores the importance of clinical correlation of adequate pathological specimens to guide IHC, as this case was initially provisionally labelled as advanced stage NSCLC, which could have precluded this patient from appropriate therapy, with significant clinical implications. Finally, this case emphasises the importance of a patient-centred approach when managing CAO.

Sarah Cullivan $\oplus^{1,5}$, John Bruzzi ${ }^{2,5}$, Teresa McHale ${ }^{3,5}$, Ramadan Shatwan ${ }^{3,5}$, Paul Donnellan ${ }^{4,5}$ and David P. Breen ${ }^{1,5}$

${ }^{1}$ Interventional Respiratory Unit, Dept of Respiratory Medicine, Galway University Hospital, Galway, Ireland. ${ }^{2}$ Dept of Radiology, Galway University Hospital, Galway, Ireland. ${ }^{3}$ Dept of Histopathology, Galway University Hospital, Galway, Ireland. ${ }^{4}$ Dept of Medical Oncology, Galway University Hospital, Galway, Ireland. ${ }^{5}$ All authors contributed equally.

Correspondence: Sarah Cullivan, Respiratory Dept, Galway University Hospital, Newcastle Road, Galway, Ireland. E-mail: sarah.cullivan@ucdconnect.ie

Received: 28 Feb 2019 | Accepted after revision: 3 Oct 2019

Conflict of interest: None declared.

\section{References}

1 Guibert N, Mazieres J, Marquette $\mathrm{CH}$, et al. Integration of interventional bronchoscopy in the management of lung cancer. Eur Respir Rev 2015; 24: 378-391.

2 Mudambi L, Miller R, Eapen GA. Malignant central airway obstruction. J Thorac Dis 2017; 9: Suppl. 10, S1087-S1110

3 Jabbardarjani $\mathrm{H}$, Herth F, Kiani A, et al. Central airway obstruction masquerading as difficult-to-treat asthma: a retrospective study. J Bronchol Interv Pulmonol 2009; 16: 6-9.

4 Kiryu T, Hoshi H, Matsui E, et al. Endotracheal/endobronchial metastases. Clinicopathologic study with special reference to developmental modes. Chest 2001; 119: 768-775.

5 Karpathiou G, Froudarakis M, Da Cruz, V et al. Endobronchial melanoma metastasis 40 years after the excision of the primary cutaneous tumor. A case report. Medicine 2017; 96: e7931.

6 Damsky WE, Rosenbaum LE, Bosenberg M. Decoding melanoma metastasis. Cancers 2010; 3: 126-163.

7 MacKie RM, Reid R, Junor B. Fatal melanoma transferred in a donated kidney 16 years after melanoma surgery. N Engl J Med 2003; 348: 567-568. 